\title{
Genetic Variation in the Androgen Receptor and Measures of Plasma Testosterone Levels Suggest Androgen Dysfunction in Alzheimer's Disease
}

Jessie S. Carr ${ }^{1}$, Luke W. Bonham ${ }^{1,2,3}$, Alicia K. Morgans ${ }^{4}$, Charles J. Ryan ${ }^{5}$, Jennifer S. Yokoyama ${ }^{1}$ and Ethan G. Geier ${ }^{1 *}$ for the Alzheimer's Disease

OPEN ACCESS

Edited by:

Tibor Hortobágyi,

University of Debrecen, Hungary

Reviewed by:

Hamid R. Sohrabi,

Macquarie University, Australia

Filippo Drago,

Università degli Studi di Catania, Italy

*Correspondence: Ethan G. Geier

Ethan.Geier@ucst:edu

specialty section:

This article was submitted to

Neurodegeneration

a section of the journal

Frontiers in Neuroscience

Received: 13 March 2018

Accepted: 13 July 2018

Published: 07 August 2018

Citation:

Carr JS, Bonham LW, Morgans AK, Ryan CJ, Yokoyama JS, and Geier EG

for the Alzheimer's Disease

Neuroimaging Initiative (2018) Genetic Variation in the Androgen Receptor and Measures of Plasma

Testosterone Levels Suggest Androgen Dysfunction in Alzheimer's

Disease. Front. Neurosci. 12:529.

doi: 10.3389/fnins.2018.00529
Neuroimaging Initiative
'Department of Neurology, Memory and Aging Center, University of California, San Francisco, San Francisco, CA,
United States, ${ }^{2}$ Department of Radiology and Biomedical Imaging, University of Callifornia, San Francisco, San Francisco,
CA, United States, ${ }^{3}$ School of Medicine, Johns Hopkins University, Baltimore, MD, United States, ${ }^{4}$ Roberr H. Lurie
Comprehensive Cancer Center, Northwestern University, Chicago, IL, United States, ${ }^{3}$ Helen Dilier Comprehensive Cancer
Center, University of California, San Francisco, San Francisco, CA, United States Comprehensive Cancer Center, Northwestern University, Chicago,
Center, University of California, San Francisco, San Francisco, CA,

Alzheimer's disease (AD) prevalence varies by sex, suggesting that sex chromosomes, sex hormones and/or their signaling could potentially modulate AD risk and progression. Low testosterone levels are reported in men with $A D$. Further, variation in the androgen receptor (AR) gene has been associated with $A D$ risk and cognitive impairment. We assessed measures of plasma testosterone levels as a biomarker of $A D$ in male participants from the Alzheimer's Disease Neuroimaging Initiative (ADNI) cohort. Baseline testosterone levels were significantly different between clinical diagnosis groups [cognitively normal controls, milld cognitive impairment (MCI), or AD], with the lowest testosterone levels in men with $A D$. Lower baseline testosterone levels were associated with higher baseline clinical severity. Change in testosterone levels between baseline and 1-year follow-up varied by diagnosis; $\mathrm{MCl}$ had the greatest decreases in testosterone leyels between baseline and 1-year follow-up. Despite differences by clinical diagnosis, there was no association between plasma testosterone and CSF biomarkers of AD pathology. We also tested single nucleotide polymorphisms (SNPS) in AR for association with $A D$ risk in a separate cohort from ADNI and found 26 SNPs associated with risk for $A D$. The top associated SNP is predicted to be an expression quantitative trait locus for $A R$ in multiple tissues, including brain, with the $A D$-associated risk allele predicted to confer lower $A R$ expression. Our findings suggest a link between the androgen pathway and $A D$ through $A \beta /$ tau independent pathways. These effects may be most pronounced during conversion from $\mathrm{MCl}$ to dementia.

Keywords: Alzheimer's disease, androgen receptor, testosterone, steroid, biomarker

\section{INTRODUCTION}

Alzheimer's disease $(\mathrm{AD})$ is a devastating neurodegenerative disorder showing sex-specific differences in prevalence and genetic risk, making sex hormones and their signaling putative disease modulators (Altmann et al., 2014). The major AD risk factor is age, and sex steroid levels decline with increasing age, precipitously for women with menopause and more gradually for men. 
While the effects of sex hormones on AD risk have been wellstudied in women (Paganini-Hill and Henderson, 1994; Espeland et al., 2004), fewer studies on sex steroids and cognitive function and/or $\mathrm{AD}$ risk exist in men.

Testosterone is the predominant androgen in men and binds to the androgen receptor (AR), regulating expression of multiple genes with diverse biological roles. AR is expressed in many brain regions, including hippocampus (Simerly et al., 1990), a region responsible for learning and memory that is heavily impacted in AD. Previous studies implicate low testosterone levels with cognitive impairment in healthy men, and higher testosterone associates with better performance in cognitive tests (Yaffe et al., 2002). Men diagnosed with mild cognitive impairment (MCI) or $\mathrm{AD}$ have lower testosterone levels compared to controls (Hogervorst et al., 2001; Paoletti et al., 2004). Low testosterone levels are also associated with greater risk of $\mathrm{AD}$ ( $\mathrm{Lv}$ et al., 2016), and precede $\mathrm{AD}$ diagnosis by 5-10 years (Moffat et al., 2004). Interestingly, brains of cognitively normal men with early AD neuropathology at autopsy have lower testosterone, suggesting lower testosterone may precede onset of clinical AD (Rosario, 2004). Low testosterone levels have also been associated with higher brain amyloid levels in MCI patients (Verdile et al., 2014). Furthermore, the prolonged use of androgen deprivation therapy (ADT) in men with prostate cancer is associated with risk of both cognitive impairment (Gonzalez et al., 2015) and AD (Nead et al., 2015, 2016).

Genetic variation within $A R$ has also been implicated in cognitive decline and risk for AD. $A R$ contains a CAG repeat expansion that correlates inversely with $A R$ transcriptional activity (Chamberlain et al., 1994). Previous studies provide conflicting results on the relationship between repeat length and cognitive decline; one found longer CAG repeats (indicating less AR activity) associated with poorer cognitive performance (Yaffe et al., 2003), another found that shorter alleles associated with increased risk of AD (Lehmann et al., 2003), and a third found no relationship between repeat length and AD risk (Ferrari et al., 2013). Exploration of genetic variation in $A R$ as a risk factor for cognitive decline and/or $\mathrm{AD}$ requires further study.

This study's goals were to assess whether plasma testosterone levels differ by clinical diagnosis and are correlated with imaging and protein biomarkers of $\mathrm{AD}$ pathology in the Alzheimer's Disease Neuroimaging Initiative (ADNI) cohort and to assess variants in $A R$ as risk factors for $\mathrm{AD}$.

\section{MATERIAL AND METHODS}

\section{Participants}

\section{Alzheimer's Disease Neuroimaging Initiative}

Alzheimer's Disease Neuroimaging Initiative is a public dataset from multiple clinical sites that includes participants with normal cognition, MCI, and AD (Weiner and Veitch, 2015). Participants are clinically diagnosed utilizing standard criteria; clinical severity was estimated using the Clinical Dementia Rating
Scale Sum of Boxes (CDR-SB) score (Morris, 1993), and all brain MRI scans were processed using FreeSurfer (Fischl, 2012). See Supplementary Methods for more detailed descriptions of ADNI's methodology. Baseline testosterone measurements were available from 188 men, 132 of whom had 1-year follow-up measurements (Supplementary Figure S1A and Supplementary Table S1). A separate cohort of age-matched, Europeanancestry men (111 AD, 98 cognitively normal controls) with whole genome sequencing (WGS) data were used in genetic analyses (Supplementary Figure S1B and Supplementary Table S2). All research participants provided written and informed consent. The UCSF institutional review board approved this study.

\section{Statistical Analyses \\ Biomarker Analyses}

Plasma testosterone measurements were collected through ADNI's Biomarkers Consortium Project ${ }^{1}$. Only pre-processed and normalized testosterone values were available from ADNI, not direct clinical plasma testosterone values. Testosterone values were transformed as deseribed in Supplementary Methods and Supplementary Figure \$2.

We assessed baseline testosterone as the outcome of interest to test for differences by the independent categorical variable of diagnosis (i.e., controls, MCI, or AD) using ANOVA. Post hoc t-tests were conducted to identify pairwise differences between diagnosis groups (i.e., controls vs. MCI; controls vs. AD; MCI vs. AD), adjusting for multiple testing via Benjamini-Hochberg $(\mathrm{BH})$ false-discovery rate (FDR) correction. We next tested whether baseline testosterone levels predict diagnosis (i.e., testosterone as a risk factor for AD) in an ordinal logistic regression framework. Similarly, we assessed testosterone as a predictor of $\mathrm{AD}$ biomarkers [structural imaging volumetrics, cerebrospinal fluid (CSF) protein levels] and clinical severity (CDR-SB) via linear regression. A second ANOVA analysis assessed differences in diagnosis using change in testosterone between baseline and 12 month follow-up. Covariates in all regression models included age, education, and $A P O E^{*} E 4$ carrier status (dichotomous). All significance tests were two-tailed and accepted as significant at $p<0.05$ after FDR correction, as relevant, and were performed in $R$.

\section{Genetic Analyses}

Low quality single nucleotide polymorphisms (SNPs) in $A R$ were removed prior to analysis (GQ $<20$ and DP $<10$ ). We also removed SNPs with low genotyping rate $(<95 \%)$ and low minor allele frequency $(\mathrm{MAF}<5 \%)$, and participants with low genotyping rates $(<90 \%)$. All statistical analyses of SNP association with $\mathrm{AD}$ risk were conducted in PLINK (Purcell et al., 2007) using $\chi^{2}$ tests and Bonferroni correction for multiple testing. Linkage disequilibrium (LD) was assessed with LDlink (Machiela and Chanock, 2015, 2017) using the 1000 Genomes Project CEU population as a reference (Auton et al., 2015).

\footnotetext{
${ }^{1}$ https://adni.loni.usc.edu/wp-content/uploads/2010/11/BC_Plasma_Proteomics_ Data_Primer.pdf
} 


\section{RESULTS}

\section{Baseline Testosterone Levels Vary by Diagnosis and Are Associated With AD}

We evaluated the relationship between plasma testosterone levels and clinical diagnosis in a subset of men in ADNI $(n=188$; Supplementary Table S1). Baseline testosterone levels were significantly different between cognitively normal controls, MCI, and $\mathrm{AD}$, with the lowest testosterone levels observed in men with $\mathrm{AD}(p=0.02)$ (Figure 1A). Post hoc pairwise tests to assess differences between specific pairs of diagnoses showed a significant difference in testosterone levels between MCI and AD ( $\left.p_{\text {Adjusted, } \mathrm{BH}}=0.015\right)$.

Given these cross-sectional differences, we assessed testosterone as a predictor of diagnosis to examine whether low testosterone levels associated with $\mathrm{AD}$. After adjusting for age, education, and $A P O E$-status, baseline testosterone levels remained associated with clinical diagnosis $(\beta=-1.41 \pm 0.57$, $p=0.014$ ), with low testosterone associated with worse diagnosis. We also stratified this cohort by $A P O E^{*} E 4$ carrier status, but did not observe statistically significant differences in baseline testosterone levels across diagnostic groups in $A P O E^{*} E 4$ noncarriers $(n=87, p=0.099)$ or $A P O E^{*} E 4$ carriers $(n=101$, $p=0.096)$.

Luteinizing hormone (LH) and sex hormone binding globulin (SHBG) modulate free testosterone levels and have been associated with cognitive function (Pike et al., 2006; Leblanc et al., 2010). However, baseline levels of LH and SHBG were not associated with diagnosis, and adding both as covariates to all regression analyses did not alter our testosterone findings.

\section{Baseline Plasma Testosterone Levels Predict Clinical Severity}

We examined whether plasma testosterone levels were associâted with clinical severity, as measured by CDR-SB. As expected, lower baseline testosterone levels were associated with higher baseline CDR-SB scores, indicating worse clinical severity $(\beta=-0.99 \pm 0.35, p=0.0069)$ (Figure 1B), and consistent with our diagnostic findings.

To further explore the relationship between plasma testosterone levels and $\mathrm{AD}$, we examined established $\mathrm{AD}$ biomarkers, including baseline brain volumetrics and CSF $\mathrm{A} \beta$ and tau. Testosterone levels were not correlated with hippocampal, entorhinal cortex, or middle temporal cortex volumes and were not correlated with CSF A $\beta$ or tau.

\section{Change in Plasma Testosterone Levels From Baseline to 12-Month Follow-Up Visit Is Largest in $\mathrm{MCl}$}

We analyzed changes in testosterone levels between baseline and 12-month follow-up and observed significant differences by diagnosis $(p=0.003$ ) (Figure 1C), with MCI versus $\mathrm{AD}$ remaining significantly different after post hoc analysis ( $\left.p_{\text {Adjusted,BH }}=0.006\right)$. The magnitude of plasma testosterone decline during the $\mathrm{MCI}$ to $\mathrm{AD}$ transition suggests that
A

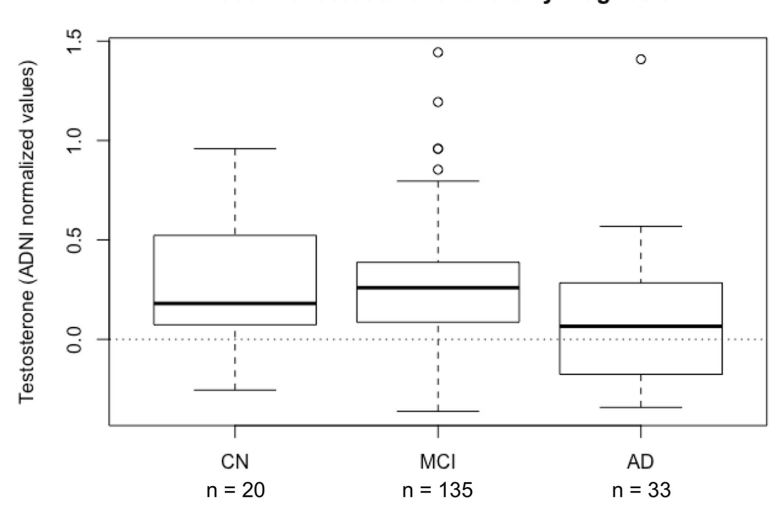

B

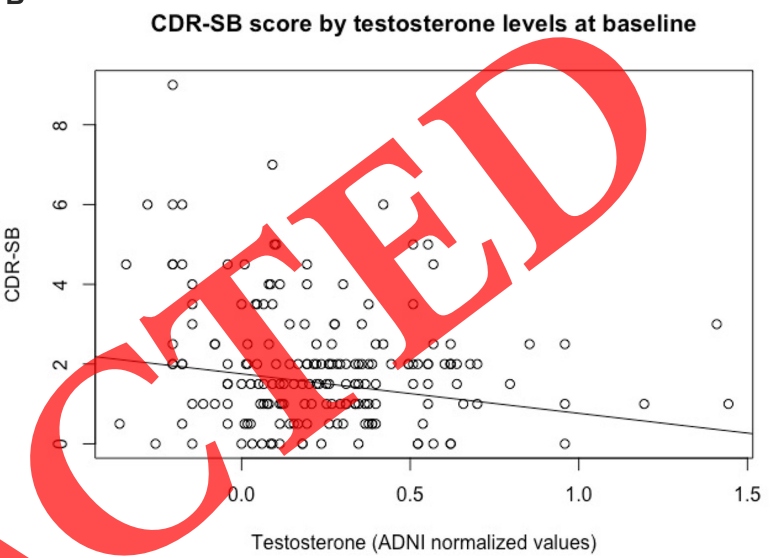

Cestosterone change (baseline to first yearly visit) by diagnosis

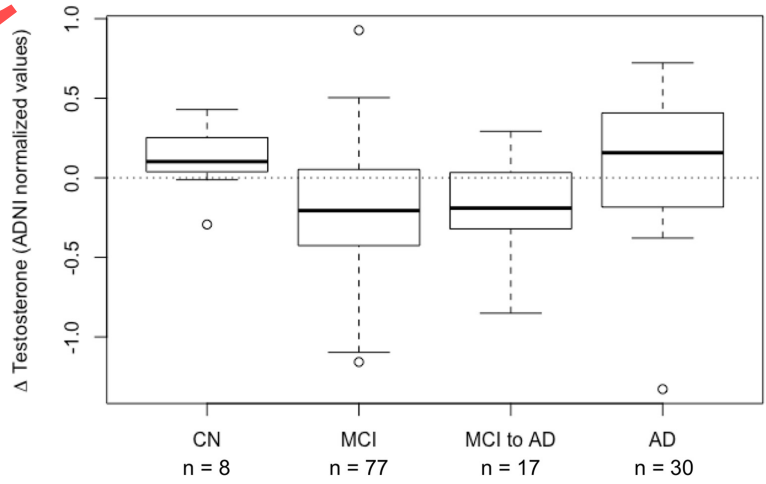

FIGURE 1 | Plasma testosterone levels are associated with diagnosis and predict clinical disease severity in men. (A) Baseline plasma testosterone levels by diagnosis in 188 male participants in ADNI. There is a significant difference between diagnostic groups of $\mathrm{CN}$ (cognitively normal controls), $\mathrm{MCl}$ (mild cognitive impairment), and AD (Alzheimer's disease) ( $p=0.02$ ), with post hoc pair-wise tests showing a significant difference in testosterone levels between $\mathrm{MCl}$ and $\mathrm{AD}$ ( $\left.\mathrm{p}_{\text {Adjusted, } \mathrm{BH}}=0.015\right)$. (B) Baseline plasma testosterone levels are a significant negative predictor of clinical severity, as measured by CDR-SB (Clinical Dementia Rating Sum of Boxes) $(\beta=-0.99 \pm 0.35$,

$p=0.007$ ). (C) Plasma testosterone changes from baseline to first annual visit by diagnosis in 132 male participants in ADNI. There is a significant difference between diagnosis groups of $\mathrm{CN}, \mathrm{MCl}, \mathrm{MCl}$ to $\mathrm{AD}$ conversion, and $\mathrm{AD}$ $(p=0.003)$. Post hoc pairwise tests show a significant difference between $\mathrm{MCl}$ and $\mathrm{AD}$ diagnosis groups ( $\left.p_{\text {Adjusted,BH }}=0.006\right)$. BH, Benjamini-Hochberg. 
testosterone levels may change most dramatically during this critical stage of disease progression.

\section{Variation in $A R$ Is Associated With AD}

Given the biological importance of testosterone's interaction with $\mathrm{AR}$ and suggested links between $\mathrm{AR}$ repeat length and cognitive impairment, we analyzed variation in $A R$ as a risk factor for $\mathrm{AD}$. We compared the MAF of 43 common SNPs across $A R$ in $111 \mathrm{AD}$ cases versus 98 cognitively normal, Europeandescent men (Supplementary Table S2 and Supplementary Figure S3). Twenty-six non-coding SNPs were associated with $\mathrm{AD}$ risk ( $\left.p_{\text {Adjusted, Bonferroni }}<0.05\right)$, with the top two SNPs found exclusively in AD cases (Figure 2A). Twenty-four of these SNPs are in LD with the top hit, rs4446868 (Figure 2B). All 43 SNPs

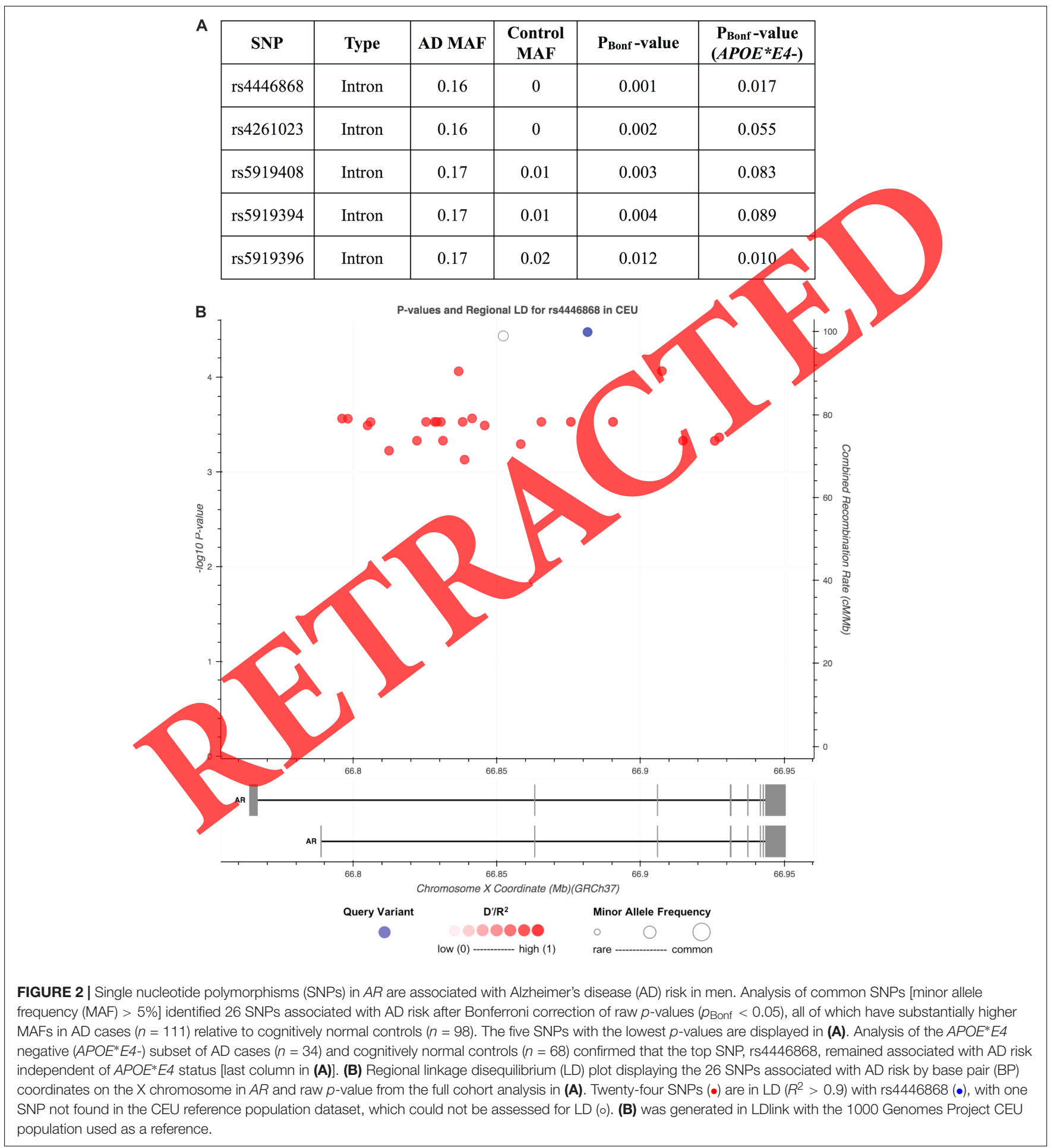


were also analyzed in the subset of participants not carrying APOE*E4 (34 cases, 68 controls). The top SNP from the full cohort analysis, rs4446868, remained associated with AD risk in $A P O E^{*} E 4$ non-carriers ( $p$ Adjusted,Bonferroni $=0.017$ ).

Since rs4446868 is intronic to $A R$, we consulted $\mathrm{GTEx}^{2}$ and Braineac $^{3}$ (Ramasamy et al., 2014) databases to examine putative functional consequences on $A R$. The rs4446868 AD risk allele is associated with lower $A R$ expression in several human brain regions, including frontal and temporal cortex (Supplementary Figure S4). This suggests genetic variants in $A R$ may contribute to $\mathrm{AD}$ risk in men possibly through reducing $A R$ expression, and is consistent with our findings that men clinically diagnosed with $\mathrm{AD}$ have lower plasma testosterone levels than other diagnostic groups.

\section{DISCUSSION}

We analyzed the relationship between measures of plasma testosterone levels and clinical diagnoses in men in the ADNI cohort. Consistent with previous studies (Hogervorst et al., 2001, 2002, 2004; Moffat et al., 2004; Paoletti et al., 2004; Watanabe et al., 2004; Lv et al., 2016), measures of baseline testosterone levels differed by clinical diagnosis, with the lowest levels in men with AD. Similarly, lower baseline testosterone measures were associated with worse clinical severity. A novel finding of our analysis is that change in testosterone levels between baseline and 12-month visit was significantly larger in MCI compared with $\mathrm{AD}$ patients. While we were only able to analyze testosterone levels over 1 year, our results are consistent with previous studies showing testosterone decreases precede AD diagnosis (Moffat et al. 2004; Rosario, 2004). The effect of testosterone decreases may therefore be most pronounced during conversion from MCI to AD, with testosterone levels "bottomed out" in AD cases. Interestingly, we did not detect a relationship between testosterone and $\mathrm{AD}$ biomarkers, including $\mathrm{CSF} A \beta$ and tau, even though previous studies found androgen levels inversely correlated with plasma A $\beta$ (Gillett et al, 2003), and brain amyloid levels as measured by $\mathrm{PiB}$ retention in MCI patients (Verdile et al., 2014). Though we are limited to clinical diagnoses of MCI and $\mathrm{AD}$ in $\mathrm{ADNI}$ based on cognitive assessments without molecular imaging or neuropathological confirmation, we observed the expected decrease in CSF $A \beta$ and increase in CSF tau in $\mathrm{MCI}$ and $\mathrm{AD}$ versus controls (ANOVA $p$-values $=2.68 \times 10^{-14}$ for $\mathrm{A} \beta$ and 0.00083 for tau; data not shown). This provides support for the underlying pathological status of our diagnostic groups and suggests that androgen dysregulation may contribute to $\mathrm{AD}$ clinical severity through mechanisms beyond neuropathology.

One limitation of our study is that raw, clinical testosterone levels were unavailable, making it impossible to back-interpolate what a given group-level difference corresponds to in specific plasma testosterone concentration values (e.g., ng/dl). Thus,

${ }^{2}$ https://www.gtexportal.org

${ }^{3} \mathrm{http}: / /$ www.braineac.org we can only make relative group-level comparisons between clinical diagnostic categories. Further, cross-sectional baseline testosterone measures did not decrease with age as expected. Future studies including men across a broader age range may help elucidate testosterone's effect on $\mathrm{AD}$ risk by age (Hogervorst et al., 2004; Rosario et al., 2011).

Our genetic analyses identified SNPs in $A R$ associated with $\mathrm{AD}$ risk, and the risk allele of the top SNP associated with lower $A R$ expression. Interestingly, this SNP retains significance in $A P O E^{*} E 4$ non-carriers, suggesting that increased risk of $\mathrm{AD}$ is independent of the effects of APOE. This supports the hypothesis that decreased androgen signaling, whether through genetic variants that decrease $A R$ expression or low plasma testosterone levels, is a risk factor for $\mathrm{AD}$, and that the androgen pathway may contribute to some of the missing heritability of $\mathrm{AD}$ risk. This hypothesis suggests that testosterone supplementation may be a viable therapeutic intervention for populations at elevated risk for cognitive decline associated with deficits in androgen signaling. We have a unique opportunity to examine these hypotheses in humans due to the purposeful suppression of androgen levels via $\mathrm{ADT}$ as a treatment for prostate cancer. A recent prospective study found that ADT exposure was associated with a significant increase in cognitive impairment by 12 months when compared to men with prostate cancer not receiving ADT and healthy controls (Gonzalez et al., 2015). Other studies suggest up to $47-69 \%$ of men on ADT experience cognitive dysfunction (Nelson et al., 2008). Additionally, two recent population-based studies suggest that risk of $\mathrm{AD}$ and dementia increases with duration of exposure to ADT (Nead al., 2015, 2016), underscoring the link between androgen deprivation and cognitive decline. The $A R$ SNPs identified here may help identify men at higher risk of cognitive impairment on ADT.

Two randomized studies have directly assessed the role of testosterone augmentation in AD/MCI. One placebocontrolled trial of $32 \mathrm{AD} / \mathrm{MCI}$ patients showed that increasing testosterone levels was associated with improved spatial memory, constructional abilities, and verbal memory (Cherrier et al., 2005). Another randomized study of 10 hypogonadal AD patients showed cognitive improvement in patients treated with testosterone (Tan and $\mathrm{Pu}, 2003$ ). However, a separate large randomized trial did not show a significant effect on cognition over 1 year of testosterone gel application in men with low testosterone and subjective memory complaints (Resnick et al., 2017). Nevertheless, our findings are in agreement with the two studies directly assessing the role of testosterone in $\mathrm{AD} / \mathrm{MCI}$, suggesting that the androgen pathway may play a role in cognitive function specifically related to $\mathrm{AD}$.

\section{ETHICS STATEMENT}

This study was carried out in accordance with the recommendations of the Human Research Protection Program Institutional Review Board (IRB). The protocol was approved by the University of California, San Francisco IRB. 


\section{AUTHOR CONTRIBUTIONS}

JC, LB, CR, JY, and EG designed the experiment. ADNI did the subject recruitment and data collection. JC, LB, and EG analyzed the data. JC, LB, AM, CR, JY, and EG did the interpretation of data/findings. JC, JY, and EG wrote the manuscript.

\section{FUNDING}

This research was supported by the National Center for Advancing Translational Sciences of the NIH under Award No. TL1 TR001871 (JC). Its contents are solely the responsibility of the authors and do not necessarily represent the official views of the NIH. Support for data analyses in this study was also provided by the Larry L. Hillblom Foundation 2016-A-005-SUP (JY), NIA K01 AG049152 (JY), the Rainwater Charitable Foundation (JY and EG), the Bluefield Project to Cure FTD (JY), the French Foundation (JY), Radiological Society of North America RMS 1741 (LB). Data collected and shared for this project was funded by the ADNI (National Institutes of Health Grant No. U01 AG024904) and DOD ADNI (Department of Defense Award No. W81XWH-12-2-0012). ADNI was funded by the National Institute on Aging, the National Institute of Biomedical Imaging and Bioengineering, and through generous contributions from the following: AbbVie, Alzheimer's Association; Alzheimer's Drug Discovery Foundation; Araclon Biotech; BioClinica, Inc.; Biogen; Bristol-Myers Squibb Company; CereSpir, Inc.; Eisai, Inc.; Elan Pharmaceuticals, Inc.; Eli Lilly and Company; EuroImmun; F Hoffmann-La Roche, Ltd. and its affiliated company Genentech,

\section{REFERENCES}

Altmann, A., Tian, L., Henderson, V. W., and Greici the APOE -related risk of developing Alzheimer disease. Ann. Neurol. 75 563-573. doi: 10.1002/ana.24135

Auton, A., Abecasis, G. R., Altshuler, D. M., Durbin, R. M., Bentley, D. R., Chakravarti, A., et al. (2015). A global reference for human genetic variation. Nature 526, 68-74. doi: 10.1038/

Chamberlain, N. L., Driver, E. D, and Miesfeld, R. L. (1994). The length and location of CAG trinucleotide repeats in the androgen receptor N-terminal domain affect transactivation function. Nucleic Acids Res. 22, 3181-3186. doi: 10.1093/nar/22.15.3181

Cherrier, M. M., Matsumoto, A. M., Amory, J. K., Asthana, S., Bremner, W., Peskind, E. R., et al. (2005). Testosterone improves spatial memory in men with Alzheimer disease and mild cognitive impairment. Neurology 64, 2063-2068. doi: 10.1212/01.WNL.0000165995.98986.F1

Espeland, M. A., Rapp, S. R., Shumaker, S. A., Brunner, R., Manson, J. E., Sherwin, B. B., et al. (2004). Conjugated equine estrogens and global cognitive function in postmenopausal women: women's health initiative memory study. JAMA 291, 2959-2968. doi: 10.1001/jama.291.24.2959

Ferrari, R., Dawoodi, S., Raju, M., Thumma, A., Hynan, L. S., Maasumi, S. H., et al. (2013). Androgen receptor gene and sex-specific Alzheimer's disease. Neurobiol. Aging 34, 2077.e19-2077.e20. doi: 10.1016/j.neurobiolaging.2013.02.017

Fischl, B. (2012). Freesurfer. Neuroimage 62, 774-781. doi: 10.1016/j.neuroimage. 2012.01.021

Gillett, M. J., Martins, R. N., Clarnette, R. M., Chubb, S. A. P., Bruce, D. G., and Yeap, B. B. (2003). Relationship between testosterone, sex hormone binding globulin and plasma amyloid beta peptide 40 in older men with subjective memory loss or dementia. J. Alzheimers Dis. 5, 267-269. doi: 10.3233/JAD2003-5401
Inc.; Fujirebio; GE Healthcare; IXICO, Ltd.; Janssen Alzheimer Immunotherapy Research \& Development, LLC; Johnson \& Johnson Pharmaceutical Research \& Development LLC; Lumosity; Lundbeck; Merck \& Co., Inc.; Meso Scale Diagnostics, LLC; NeuroRx Research; Neurotrack Technologies; Novartis Pharmaceuticals Corporation; Pfizer, Inc.; Piramal Imaging; Servier; Takeda Pharmaceutical Company; and Transition Therapeutics. The Canadian Institutes of Health Research provides funds to support ADNI clinical sites in Canada. Private sector contributions are facilitated by the Foundation for the National Institutes of Health (www.fnih.org). The grantee organization is the Northern California Institute for Research and Education, and the study is coordinated by the Alzheimer's Disease Cooperative Study at the University of California, San Diego. ADNI data are disseminated by the Laboratory for Neuro Imaging at the University of Southern California.

\section{ACKNOWLEDGMENTS}

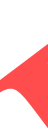

We thank the ADNI research participants for their longstanding
dedication.

\section{SUPPLEMENTARY MATERIAL}

The Supplementary Material for this article can be found online at: https://www.frontiersin.org/articles/10.3389/fnins. 2018.00529/full\#supplementary-material

Gonzalez, B. D., Jim, H. S. L., Booth-Jones, M., Small, B. J., Sutton, S. K., Lin, H. Y., et al. (2015). Course and predictors of cognitive function in patients with prostate cancer receiving androgen-deprivation therapy: a controlled comparison. J. Clin. Oncol. 33, 2021-2027. doi: 10.1200/JCO.2014.60.1963

Hogervorst, E., Bandelow, S., Combrinck, M., and Smith, A. D. (2004). Low free testosterone is an independent risk factor for Alzheimer's disease. Exp. Gerontol. 39, 1633-1639. doi: 10.1016/j.exger.2004.06.019

Hogervorst, E., Lehmann, D. J., Warden, D. R., McBroom, J., and Smith, A. D. (2002). Apolipoprotein E epsilon4 and testosterone interact in the risk of Alzheimer's disease in men. Int. J. Geriatr. Psychiatry 17, 938-940. doi: 10.1002/ gps.714

Hogervorst, E., Williams, J., Budge, M., Barnetson, L., Combrinck, M., and Smith, A. D. (2001). Serum total testosterone is lower in men with Alzheimer's disease. Neuroendocrinol. Lett. 22, 163-168.

Leblanc, E. S., Wang, P. Y., Janowsky, J. S., Neiss, M. B., Fink, H. A., Yaffe, K., et al. (2010). Association between sex steroids and cognition in elderly men. Clin. Endocrinol. 72, 393-403. doi: 10.1111/j.1365-2265.2009.03692.x

Lehmann, D. J., Butler, H. T., Warden, D. R., Combrinck, M., King, E., Nicoll, J. A. R., et al. (2003). Association of the androgen receptor CAG repeat polymorphism with Alzheimer's disease in men. Neurosci. Lett. 340, 87-90. doi: 10.1016/S0304-3940(03)00069-7

Lv, W., Du, N., Liu, Y., Fan, X., Wang, Y., Jia, X., et al. (2016). Low testosterone level and risk of Alzheimer's disease in the elderly men: a systematic review and meta-analysis. Mol. Neurobiol. 53, 2679-2684. doi: 10.1007/s12035-015-9315-y

Machiela, M. J., and Chanock, S. J. (2015). LDlink: a web-based application for exploring population-specific haplotype structure and linking correlated alleles of possible functional variants. Bioinformatics 31, 3555-3557. doi: 10.1093/ bioinformatics/btv402

Machiela, M. J., and Chanock, S. J. (2017). LDassoc: an online tool for interactively exploring genome-wide association study results and prioritizing 
variants for functional investigation. Bioinformatics 34, 887-889. doi: 10.1093/ bioinformatics/btx561

Moffat, S. D., Zonderman, A. B., Metter, E. J., Kawas, C., Blackman, M. R., Harman, S. M., et al. (2004). Free testosterone and risk for Alzheimer disease in older men. Neurology 62, 188-193. doi: 10.1212/WNL.62.2.188

Morris, J. C. (1993). The clinical dementia rating (CDR): current version and scoring rules. Neurology 43, 2412-2414. doi: 10.1212/WNL.43.11.2412-a

Nead, K. T., Gaskin, G., Chester, C., Swisher-McClure, S., Leeper, N. J., and Shah, N. H. (2015). Androgen deprivation therapy and future Alzheimer's Disease risk. J. Clin. Oncol. 34, 566-571. doi: 10.1200/JCO.2015.63.6266

Nead, K. T., Gaskin, G., Chester, C., Swisher-McClure, S., Leeper, N. J., and Shah, N. H. (2016). Association between androgen deprivation therapy and risk of dementia. JAMA Oncol. 19104, 1-7. doi: 10.1001/jamaoncol.2016.3662

Nelson, C. J., Lee, J. S., Gamboa, M. C., and Roth, A. J. (2008). Cognitive effects of hormone therapy in men with prostate cancer: a review. Cancer 113, 1097-1106. doi: 10.1002/cncr.23658

Paganini-Hill, A., and Henderson, V. W. (1994). Estrogen deficiency and risk of Alzheimer's disease in women. Am. J. Epidemiol. 140, 256-261. doi: 10.1093/ oxfordjournals.aje.a117244

Paoletti, A. M., Congia, S., Lello, S., Tedde, D., Orrù, M., Pistis, M., et al. (2004). Low androgenization index in elderly women and elderly men with Alzheimer's disease. Neurology 62, 301-303. doi: 10.1212/01.WNL.0000094199.60829.F5

Pike, C. J., Rosario, E. R., and Nguyen, T. V. (2006). Androgens, aging, and Alzheimer's disease. Endocrine 29, 233-241. doi: 10.1385/ENDO:29:2:233

Purcell, S., Neale, B., Todd-Brown, K., Thomas, L., Ferreira, M. A. R., Bender, D., et al. (2007). PLINK: a Tool Set for whole-genome association and populationbased linkage analyses. Am. J. Hum. Genet. 81, 559-575. doi: 10.1086/519795

Ramasamy, A., Trabzuni, D., Guelfi, S., Varghese, V., Smith, C., Walker, R., et al. (2014). Genetic variability in the regulation of gene expression in ten regions of the human brain. Nat. Neurosci. 17, 1418-1428. doi: 10.1038/nn.3801

Resnick, S. M., Matsumoto, A. M., Stephens-Shields, A. J., Ellenberg, S. S., Gill, T. M., Shumaker, S. A., et al. (2017). Testosterone treatment and cognitive function in older men with low testosterone and age-associated memory impairment. JAMA 317, 717-727. doi: 10.1001/jama.2016.21044

Rosario, E. R. (2004). Age-Related testosterone depletion and the development of Alzheimer Disease. J. Am. Med. Assoc. 292, 1431-1432. doi: 10.1001/jama.292. 12.1431-b

Rosario, E. R., Chang, L., Head, E. H., Stanczyk, F. Z., and Pike, C. J. (2011). Brain levels of sex steroid hormones in men and women during hormal aging and in Alzheimer's disease. Neurobiol. Aging 32,604-613. doi: $10.1016 /$ j.neurobiolaging.2009.04.008

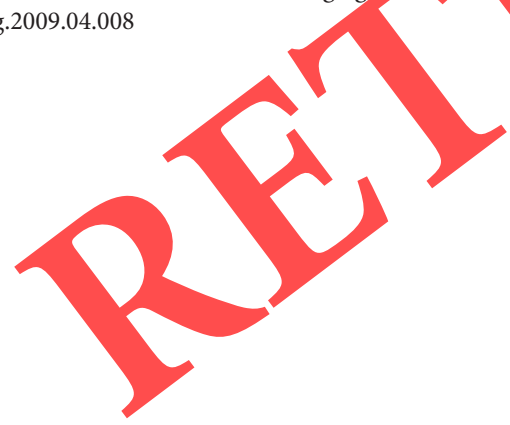

Simerly, R. B., Swanson, L. W., Chang, C., and Muramatsu, M. (1990). Distribution of androgen and estrogen receptor mRNA-containing cells in the rat brain: an in situ hybridization study. J. Comp. Neurol. 294, 76-95. doi: 10.1002/cne. 902940107

Tan, R. S., and Pu, S. J. (2003). A pilot study on the effects of testosterone in hypogonadal aging male patients with Alzheimer's disease. Aging Male 6, 13-17. doi: 10.1080/tam.6.1.13.17

Verdile, G., Laws, S. M., Henley, D., Ames, D., Bush, A. I., Ellis, K. A., et al. (2014). Associations between gonadotropins, testosterone and $\beta$ amyloid in men at risk of Alzheimer's disease. Mol. Psychiatry 19, 69-75. doi: 10.1038/mp.2012.147

Watanabe, T., Koba, S., Kawamura, M., Itokawa, M., Idei, T., Nakagawa, Y., et al. (2004). Small dense low-density lipoprotein and carotid atherosclerosis in relation to vascular dementia. Metabolism. 53, 476-482. doi: 10.1016/j.metabol. 2003.11.020

Weiner, M. W., and Veitch, D. P. (2015). Introduction to special issue: overview of Alzheimer's disease neuroimaging initiative. Alzheimers Dement. 11, 730-733. doi: 10.1016/j.jalz.2015.05.007

Yaffe, K., Edwards, E. R., Lui, L.-Y., Zmuda, J. M., Ferrell, R. E., and Cauley, J. A. (2003). Androgen receptor CAG repeat polymorphism is associated with cognitive function in older men. Biol. Psychiatry 54, 943-946. doi: 10.1016/ S0006-3223(03)00115-X

Yaffe, K., Lui, L.-Y., Zmuda, J., and Cauley, J (2002). Sex hormones and cognitive function in older men. J. Am. Geriatr. Soc. 50, 707-712. doi: 10.1046/j.15325415.2002.50166.x

Conflict of Interest Statement: AM has received honoraria from Sanofi, AstraZeneca, Genentech, Inc., and Janssen Pharmaceuticals, Inc. CR has received honoraria from Janssen Pharmaceuticals, Inc. andBayer AG.

The remaining atthors declare that the research was conducted in the absence of any commercial or financial relationships that could be construed as a potential conflict of interest.

Copyright @ 2018 Carr, Bonham, Morgans, Ryan, Yokoyama, and Geier for the Alzheimer's Disease Neuroimaging Initiative. This is an open-access article distributed under the terms of the Creative Commons Attribution License (CC BY). The use, distribution or reproduction in other forums is permitted, provided the original author(s) and the copyright owner(s) are credited and that the original publication in this journal is cited, in accordance with accepted academic practice. No use, distribution or reproduction is permitted which does not comply with these terms. 